\title{
Multi-Scale Turbulence Model in Simulation of Supersonic Crossflow Part 2: Inclined Injection
}

\author{
Ez Hassan $^{*}$ and Hikaru Aono ${ }^{\dagger}$ \\ University of Michigan, Ann Arbor, MI \\ John Boles \\ Taitech, Inc., Beavercreek, $\mathrm{OH}$ \\ Douglas Davis ${ }^{\S}$ \\ Air Force Research Laboratory, Fairborn, $\mathrm{OH}$ \\ Wei Shyy ${ }^{*}{ }^{* \dagger}$ \\ University of Michigan, Ann Arbor, MI \\ The Hong Kong University of Science and Technology, Kowloon, Hong Kong
}

\begin{abstract}
The multi-scale turbulence approach is useful in predicting mean flows in problems containing complex turbulent structures that are otherwise unattainable using standard Reynolds-averaged Navier-Stokes models. In crossflow simulations using the multi-scale turbulence approach, modifying the sub-filter turbulent mass diffusion based on the resolved field was useful in simulation of normal injection with coarser grids. Inclined injection angles are commonly evaluated for improved mixing performance; this work is therefore aimed at assessing the multi-scale approach with inclined injection in supersonic crossflow. Reynolds-averaged Navier-Stokes and multi-scale without the adaptive turbulent Schmidt number approach are compared to experimental fuel concentration measurements. Unlike previous normal injection results, multiscale alone did not improve the results significantly even with a grid of nearly 30 million cells. Turbulent fluctuations of the normal and inclined injections are compared showing vortical structures that are scattered and of higher frequency in the latter case. The adaptive approach is then used with the same grid in the inclined case in order to improve the prediction of turbulent mixing. Results with the adaptive turbulent Schmidt number approach are superior to both RANS and multi-scale alone when compared to experimental predictions.
\end{abstract}

\footnotetext{
* PhD Candidate, Aerospace Engineering Department

${ }^{\dagger}$ Postdoctoral Research Fellow, Aerospace Engineering Department, currently research scientist, ISAS/JAXA, Japan

* Research Scientist, AFRL/RZAS

$\S$ Aerospace Engineer, AFRL/RZAS

** Clarence L. "Kelly" Johnson Collegiate Professor, Aerospace Engineering Department

${ }^{\dagger}$ Chair Professor, Mechanical Engineering at HKUST

American Institute of Aeronautics and Astronautics
} 


\section{Nomenclature}

\begin{tabular}{|c|c|}
\hline$C_{p}$ & $=$ heat capacity at constant pressure \\
\hline$C_{g s}$ & $=$ generalized coefficient for numerical viscosity estimation \\
\hline$C_{s}$ & $=$ Smagorinsky constant \\
\hline$D$ & $=$ injector inner diameter \\
\hline$D_{i_{m}}$ & $=$ diffusion vector for species $m$ \\
\hline$E$ & $=$ total specific energy of the mixture \\
\hline$H$ & $=$ total specific enthalpy of the mixture \\
\hline$F_{2}$ & $=$ second blending function, Menter SST \\
\hline$f_{d}$ & $=$ filter function \\
\hline$h_{m}$ & $=$ species specific enthalpy \\
\hline$k$ & $=$ turbulent specific kinetic energy \\
\hline$L_{G}$ & $=$ length scale based on cell size \\
\hline$L_{T}$ & $=$ measure of turbulence length scale \\
\hline NS & $=$ total number of species \\
\hline$p$ & $=$ pressure \\
\hline$P r_{t}$ & $=$ turbulent Prandtl number \\
\hline$q$ & $=$ Injection momentum ratio \\
\hline$q_{i}$ & $=$ heat transfer vector \\
\hline$S_{i j}(\tilde{u})$ & $=$ strain rate tensor of Favre-averaged subgrid RANS solution \\
\hline$S c_{t}$ & $=$ turbulent Schmidt number \\
\hline$T$ & $=$ mixture temperature \\
\hline$u_{i}$ & $=$ mixture velocity \\
\hline$Y_{m}$ & $=$ mole fraction of species $\mathrm{m}$ \\
\hline$\mu_{t}$ & $=$ dynamic eddy viscosity \\
\hline$\mu_{\text {num }}$ & $=$ dynamic numerical viscosity \\
\hline$v_{t}$ & $=$ kinematic eddy viscosity \\
\hline $\bar{\omega}$ & $=$ average chemistry source term \\
\hline$\rho$ & $=$ mixture density \\
\hline$\tau_{i j}$ & $=$ stress tensor \\
\hline$(\sim)$ & $=$ Favre average operator \\
\hline$(-)$ & $=$ time average operator \\
\hline & $=$ superscript for Reynolds terms \\
\hline Res & $=$ subscript for quantities calculated from resolved field \\
\hline $\mathrm{R}$ & superscript for fluctuations in the resolved portion of the field \\
\hline
\end{tabular}

\section{Introduction}

CUPERSONIC crossflow analysis is needed to understand the physics behind supersonic combustion occurring in scramjet engines. Because the residence time of the flow in the combustor is often on the order of chemical time scales, it is of utmost importance for the fuel and oxidizer to be mixed quickly. It is necessary to gain better understanding of the effect of different fuel injection configuration and combustor geometries on the mixing process in order to achieve desirable designs for scramjet engines.

Reynolds-averaged Navier-Stokes (RANS) based approaches have shown some success in understanding the mechanics of supersonic cross flows. Tam et al. ${ }^{1}$ used RANS based methods with Menter Shear Stress Transport $\left(\mathrm{SST}^{2}\right)$, Menter baseline $\left(\mathrm{BSL}^{2}\right)$, and Wilcox $k-\omega^{3}$ turbulence models to simulate the experiments of Gruber et al. ${ }^{4-6}$. It was shown that the Wilcox $k-\omega$ model performed the best of all three; however, in all the models tested the fuel penetration height was over predicted by up to $25 \%$. Palekar et al. $^{7}$ obtained better correlations with penetration heights with a 4.7 million cell grid using the commercial computational fluid dynamics (CFD) code GASP. The Wilcox $k-\omega$ turbulence model was also utilized for turbulence closure. Coarser grids did not sufficiently resolve the flow and spanwise fuel penetration significantly deviated from the experiment. Maddalena et al. ${ }^{8}$ used the $k-\omega$ Wilcox turbulence model to simulate an aeroramp injection scheme as well as transverse injection of sonic helium 
into air. Total pressure loss for transverse injection was shown to be greater than of normal injection configuration with the computational results not correlating well with the experiment.

As stated by many researchers ${ }^{9-11}$, there are some obvious limitations to RANS models when applied to unsteady problems because they tend to be overly dissipative (predicting higher eddy viscosity and damping the unsteady motion of the fluid). The crossflow problem usually involves large scale unsteady turbulent structures, density gradients, and shock boundary layer interactions as evident by experimental findings ${ }^{4-6}$. These phenomena are difficult to capture correctly with standard RANS approaches and may benefit from methods capable of resolving turbulent structures such as Direct Numerical Simulation (DNS) or Large Eddy Simulation (LES). LES is capable of capturing the large scale turbulent structures, and while not as computationally expensive as DNS, it is impractical for use in the supersonic crossflow problem because a prohibitively large number of grid points must be used to resolve the boundary layer. Recently, Kawai and Lele ${ }^{12}$ conducted a LES of sonic injection into a supersonic crossflow and showed key physics of the jet mixing in supersonic crossflow such as clockwise and counterclockwise rotating strong counter-rotating vortices, a pair of U-shaped counter rotating vortices. To reduce the expense of the computations, the Reynolds number was lowered by a factor of six, relative to the experiment ${ }^{13}$ but the boundary layer thickness upstream of jet injection was matched.

Detached eddy simulation (DES) ${ }^{11}$ and hybrid RANS/LES methods, where RANS is used at the wall boundary and LES is used elsewhere, has shown a great promise in the solution of supersonic crossflow problem.

Peterson et al. ${ }^{14}$ used a DES model based on Spalart-Allmaras one equation turbulence model to simulate supersonic cross flow experiments conducted at Virginia Polytechnic University. DES compared favorably to the experiment with results superior to RANS. Boles et al. ${ }^{15-17}$ simulated Gruber et al. ${ }^{4-6}$ air and helium injection cases as well as ethylene injection case conducted at Air Force Research Laboratory (AFRL) and reported by Lin et al. ${ }^{18}$. In all cases time averaged hybrid results were superior to RANS quantitatively and qualitatively when compared to experimental injectant distribution.

In an effort similar to hybrid RANS/LES, Hassan et al. ${ }^{19}$ used the multi-scale turbulence approach to reduce the eddy viscosity in SST turbulence model ${ }^{2}$. They were able to capture large scale turbulent structures showing remarkable improvement in the simulation of Lin et al. ${ }^{18}$ over the RANS approach. Grid refinement studies on adapted unstructured grids showed that very fine grids are needed to resolve enough turbulent scales to reach any form of grid independence. This was probably due to the use of the dissipative second-order upwind schemes. It was also shown that RANS simulations are very sensitive to the turbulent Schmidt number $\left(S c_{t}\right)^{19,20}$. Hassan et al. ${ }^{19}$ and Boles et al. ${ }^{17}$ derived estimates for the turbulent Schmidt number based on the resolved field which was not constant with strong variations throughout the flow.

There have been many efforts in the RANS community to calculate, rather than specify, the turbulent Prandtl and Schmidt numbers as early as $1975^{21}$. Methods based on the mixing length used a two equation model to calculate turbulent diffusivity in conjunction with $k-\varepsilon^{22,}{ }^{23}$. In general, the results from these methods showed an improvement over $k-\varepsilon$ alone in low temperature high Mach number cases. Guo et al. ${ }^{24}$ used a genetic algorithm to obtain model constants for a diffusion vector transport equation used in addition to $k-\varepsilon$. The results showed some improvement over a baseline $k-\varepsilon$ model for a jet-in-crossflow application. CRAFT Tech developed a variable $\operatorname{Prandtl}\left(P r_{t}\right)$ and $\operatorname{Schmidt}\left(S c_{t}\right)$ number approach based largely on earlier efforts ${ }^{22}{ }^{23}$ with added compressibility correction. The model showed improvements over a constant $P r_{t} / S c_{t}$ in a range of classical validation cases ${ }^{25}{ }^{26}$.

Keistler ${ }^{27}$ used a reacting model with variable $\operatorname{Pr}_{t} / S c_{t}$ method designed for high speed flows and based on CRAFT tech efforts ${ }^{25,26}$ to simulate Lin et al. ${ }^{18}$ mixing case. The results were compared to those obtained by hybrid RANS/LES method of Boles et al. ${ }^{17}$ While the variables approach showed some limited improvement over RANS in predicting fuel concentration levels. It fell short to the hybrid RANS/LES method in predicting jet shape mainly due to inability to produce large scale turbulent structures.

Hassan et al. ${ }^{28}$ then proposed an extension to their earlier multi-scale approach ${ }^{19}$ allowing for the calculation of the turbulent Schmidt number based on the resolved field. The proposed method does not utilize transport equations with ad hoc constants. The value of the turbulent Schmidt number in the sub-filter RANS model is adaptively changed based on the resolved turbulent field. At every time step, the average turbulent Schmidt number based on the ratio of the resolved mass and momentum eddy viscosity is calculated. This value is used in the mass transport equation instead of the specified constant value.

In previous efforts ${ }^{19,28}$, the test cases considered were normal injections of sonic ethylene into Mach 2 air based on experiments conducted at AFRL and reported by Lin et al. ${ }^{18}$ Because the Injection was conducted at a 90 degree angle, large separation between the jet and crossflow occurred causing violent breakup and large scale turbulent structures. In this effort we focus on 30 degree injection with momentum ratio, $\mathrm{q}=0.5$. A grid of 29 million cells is used with the multi-scale method while a grid of 6 million cells is used with the RANS approach. Adaptive $\mathrm{Sc}_{\mathrm{t}}$ is 
used optionally and all results are compared to experimental measurements and variance. The dynamics of turbulent structures are compared to those obtained with the 90 degree injection in previous effort ${ }^{19}$.

\section{Governing Equations and Computational Modeling Approaches}

A density-based, finite volume code, Loci-Chem ${ }^{29,30}$, is utilized in this study. The code is capable of handling mixed element type unstructured grids. The convective fluxes are based on Roe's flux difference splitting ${ }^{31}$. Both convective and diffusive fluxes are evaluated to second order accuracy. Menter SST ${ }^{2}$ model is used for turbulence closure along with multi-scale treatment.

\section{Multi-Scale Turbulence Treatment:}

With the concept of eddy viscosity used in either a standard two-equation RANS approach or the Smagorinsky subgrid model in LES, both filtered and averaged mass, momentum, and energy equations yield an identical mathematical form as indicated by Germano ${ }^{32}$,

$$
\begin{gathered}
\frac{\partial}{\partial t}\left(\bar{\rho} \tilde{Y}_{m}\right)+\frac{\partial}{\partial x_{i}}\left(\bar{\rho} \tilde{Y}_{m} \tilde{u}_{i}\right)=\frac{\partial}{\partial x_{i}}\left(D_{i_{m}}+D_{i_{m}}^{R}\right)+\overline{\dot{\omega}} \\
\frac{\partial}{\partial t}\left(\bar{\rho} \tilde{u}_{i}\right)+\frac{\partial}{\partial x_{j}}\left(\bar{\rho} \tilde{u}_{i} \tilde{u}_{j}\right)=-\frac{\partial \bar{p}}{\partial x_{j}}+\frac{\partial}{\partial x_{j}}\left(\tau_{i j}+\tau_{i j}^{R}\right) \\
\frac{\partial}{\partial t}(\bar{\rho} \tilde{E})+\frac{\partial}{\partial x_{j}}\left(\bar{\rho} \widetilde{H} \tilde{u}_{j}\right)=\frac{\partial}{\partial x_{j}}\left[\tilde{u}_{j}\left(\tau_{i j}+\tau_{i j}^{R}\right)-\left(q_{j}+q_{j}^{R}\right)\right]
\end{gathered}
$$

where the Reynolds terms are modeled as follows:

$$
\begin{gathered}
D_{i_{m}}^{R} \approx-\frac{\mu_{t}}{S c_{t}} \frac{\partial \tilde{Y}_{m}}{\partial x_{j}} \\
\tau_{i j}^{R} \approx \mu_{t}\left(\frac{\partial \tilde{u}_{i}}{\partial x_{j}}+\frac{\partial \tilde{u}_{j}}{\partial x_{i}}-\frac{2}{3} \frac{\partial \tilde{u}_{k}}{\partial x_{k}} \delta_{i j}\right) \\
q_{j}^{R} \approx-\frac{\mu_{t}}{P r_{t}} \frac{\partial \tilde{T}}{\partial x_{j}}-\sum_{m=1}^{N S} h_{m} \frac{\mu_{t}}{S c_{t}} \frac{\partial \tilde{Y}_{m}}{\partial x_{j}}
\end{gathered}
$$

The variables in Eqs. (1) and (2) are shown in the filtered form, however they could represent averaged quantities in which case the value of the eddy viscosity would be produced via a two equation turbulence model. They could also be instantaneous variables when the eddy viscosity is set to zero. When they are filtered, a Smagorinsky subgrid eddy viscosity is defined as follows:

$$
\mu_{t}=\bar{\rho} C_{s} L_{G}^{2} S
$$




$$
S=\sqrt{\frac{\partial \tilde{u}_{i}}{\partial x_{j}} \frac{\partial \tilde{u}_{j}}{\partial x_{i}}+\frac{\partial \tilde{u}_{i}}{\partial x_{j}} \frac{\partial \tilde{u}_{i}}{\partial x_{j}}-\frac{2}{3}\left(\frac{\partial \tilde{u}_{i}}{\partial x_{i}}\right)^{2}}
$$

Assuming that we average the result of each model, Eqs. (1) and (2) combined with the averaging procedure, become a single mathematical formulation in which we input an eddy viscosity and output averaged quantities. This formulation is valid for averaged, filtered and instantaneous equations as long as the correct eddy viscosity value is provided.

In multi-scale modeling, we assume the input eddy viscosity is a continuous function that varies from the RANS value to zero depending on the ratio of the grid size, $L_{G}$, to a locally defined turbulence length scale, $L_{T}$. After the appropriate eddy viscosity is found it is used in Eqs. (1) and (2). The multi-scale model allows smooth transition from RANS to LES to DNS with the grid size and local turbulent length scales being the determining factors of which model to use. When employing this approach, there are no limitations on grid size or geometry because the transition between the models is allowed to occur anywhere in the computational domain.

The multi-scale treatment can be implemented easily into any RANS code as a mere modification to the eddy viscosity that is output from the two equation model, before using it in the mass, momentum and energy transport. This modification depends on the definition of the filter function, the turbulence length scale $\left(L_{T}\right)$ and the multiscale eddy viscosity.

\section{Multi-scale approach}

A multi-scale turbulence approach proposed by Hassan et al. ${ }^{19}$ is used in this effort. It is based on a filter function that is a blend between the filter-based model (FBM) developed by Johansen et al. ${ }^{33}$ and the multi-scale hybrid RANS/LES turbulence model developed by Nichols and Nelson ${ }^{34}$. The turbulence length scale, $L_{T}$, is defined as follows:

$$
L_{T}=\frac{\sqrt{k}}{C_{4} \max \left(\omega, S F_{2} / a_{1}\right)}
$$

where,

$$
C_{4}=\frac{C_{s}}{\sqrt{0.3}}
$$

and the eddy viscosity defined as,

$$
v_{t}=v_{t_{R A N S}} f_{d}
$$

The constant, $a_{1}$, is the same one used in SST model ${ }^{2}$. The value of $C_{s}$ varies, but in this approach we use the value of 0.01 as recommended by Boles ${ }^{17}$ for ethylene injection cases. The grid length scale length scale, $L_{G}$, is defined as twice the maximum distance between the cell center and each face as follow

$$
L_{G}=2 \max \left|\vec{X}_{\text {center }}-\vec{X}_{\text {face }}\right|
$$

Finally the filter function takes the form adopted in Hassan et al. ${ }^{19}$, 


$$
f_{d}=\frac{1}{2}\left\{1+\tanh \left[2 \pi\left(\frac{1}{1+\left(\frac{L_{T}}{2 L_{G}}\right)^{4 / 3}}-0.5\right)\right]\right\}+0.0622
$$

\section{Adaptive turbulent Schmidt number approach}

In standard RANS approach the turbulent momentum flux is modeled as follows,

$$
\begin{gathered}
-\overline{\rho u_{\imath}^{\prime} u_{\jmath}^{\prime}}=\mu_{t} S_{i j}\left(\tilde{u}_{i}\right)-\frac{2}{3} \overline{\rho k} \delta_{i j} \\
S_{i j}\left(\tilde{u}_{i}\right)=\left(\frac{\partial \tilde{u}_{i}}{\partial x_{j}}+\frac{\partial \tilde{u}_{j}}{\partial x_{i}}-\frac{2}{3} \frac{\partial \tilde{u}_{k}}{\partial x_{k}} \delta_{i j}\right)
\end{gathered}
$$

Turbulent mass flux for multispecies is modeled as,

$$
-\overline{\rho u_{l}^{\prime} Y_{m}^{\prime}}=\frac{\mu_{t}}{S c_{t}} \frac{\partial \tilde{Y}_{m}}{\partial x_{i}}
$$

We can multiply Eq. (9) by $S_{i j}\left(\tilde{u}_{i}\right)$ and Eq. (10) by $\partial \tilde{Y}_{m} / \partial x_{i}$ to obtain scalar equations. Then we can define mass and momentum eddy viscosities based on turbulent fluctuations,

$$
\begin{gathered}
v_{t, m o m}=\frac{\mu_{t}}{\bar{\rho}}=-\frac{\left(\overline{\rho u_{\imath}^{\prime} u_{\jmath}^{\prime}}-\frac{2}{3} \overline{\rho k} \delta_{i j}\right) S_{i j}\left(\tilde{u}_{i}\right)}{\bar{\rho} S_{i j}\left(\tilde{u}_{i}\right) S_{i j}\left(\tilde{u}_{i}\right)} \\
v_{t, m a s s}=\frac{\mu_{t}}{S c_{t} \bar{\rho}}=-\frac{\left(\overline{\rho u_{l}^{\prime} Y_{m}^{\prime}}\right)}{\bar{\rho}} \frac{\left(\partial \tilde{Y}_{m} / \partial x_{i}\right)}{\left(\partial \tilde{Y}_{m} / \partial x_{i}\right)\left(\partial \tilde{Y}_{m} / \partial x_{i}\right)}
\end{gathered}
$$

The turbulent Schmidt number can be obtained by dividing the mass and momentum eddy viscosities. When using the multi-scale model approach only fluctuations in the resolved field are calculated directly, while those in the sub-filter field are calculated using the RANS model. To eliminate uncertainties associated with specifications of the turbulent Schmidt number. Hassan et al. ${ }^{28}$ defined the resolved turbulent Schmidt number based on resolved quantities as follows,

$$
S c_{t_{-} R e s}=\frac{\frac{\left(\overline{\rho u_{l}^{R} u_{j}^{R}}-\frac{2}{3} \overline{\rho k_{R e s}} \delta_{i j}\right) S_{i j}\left(\tilde{u}_{i}\right)}{\bar{\rho} S_{i j}\left(\tilde{u}_{i}\right) S_{i j}\left(\tilde{u}_{i}\right)}}{\frac{\left(\overline{\rho u_{l}^{R} Y_{m}^{R}}\right)}{\bar{\rho}} \frac{\left(\partial \tilde{Y}_{m} / \partial x_{i}\right)}{\left(\partial \tilde{Y}_{m} / \partial x_{i}\right)\left(\partial \tilde{Y}_{m} / \partial x_{i}\right)}}
$$


The use of the resolved turbulent Schmidt number in Eq. (12) the RANS model assumes that the ratio of turbulent momentum fluxes and turbulent mass fluxes in the resolved portion of the flow is the same as in the unresolved portions of the flow. In other words if we split the energy spectrum at a certain wave length equal to the filter function we have equal ratios of mass and momentum turbulent fluxes in the sub-filter and resolved portions. This assumption was tested by Hassan et al. ${ }^{28}$ and it was found that the effect of the adaptive approach was limited because numerical viscosity was not taken into consideration when calculating sub-filter turbulent diffusion. Hassan et al. ${ }^{28}$ suggested the following correction to Reynolds diffusion term,

$$
D_{i_{m}}^{R} \approx-\frac{\mu_{t}+\mu_{\mathrm{num}}}{S c_{t}} \frac{\partial \tilde{Y}_{m}}{\partial x_{j}}
$$

The numerical viscosity is estimated using the generalized coefficient approach ${ }^{35,36}$ taking the following approximate value

$$
\mu_{\mathrm{num}}=\bar{\rho} C_{\mathrm{gs}} L_{G}^{2}|S|
$$

A generalized coefficient, $C_{\mathrm{gs}}$, value of 0.2 is used in this effort as suggested by Mossi et al. ${ }^{36}$ for a second order scheme in a compressible fully developed flow.

The resolved turbulent Schmidt number $\left(S c_{t_{-} R e s}\right)$ is calculated at every time step during the simulation and is used calculating RANS turbulent mass diffusion in Eq. (2). The value of the resolved turbulent Schmidt number is theoretically constant at every time step however, because averages are not available a priori, the estimations of the averages improve as the simulation proceeds and $S c_{t_{-} R e s}$ converge to a constant value. Also the application of $S c_{t_{-} R e s}$ into the RANS sub-filter model is not done until a number of iterations at constant $S c_{t}$ to avoid erroneous values of $S c_{t_{-} R e s}$ at the beginning of the simulation. Also the positivity of the resolved turbulent Schmidt number is maintained by taking the absolute value.

\section{Experimental setup}

Lin et al. ${ }^{18}$ performed a sonic injection of ethylene into Mach 2 air crossflow at the continuous flow supersonic tunnel at Wright-Patterson Air Force Base, Ohio. The tunnel has a constant test area of $131 \times 152 \mathrm{~mm}$. The injectors were circular with different diameters, injection angles, and momentum ratios. In this study we focus on the case with $D=0.1875$ inches $(4.76 \mathrm{~mm})$ inclined 30 degree injection with a momentum ratio of 0.5 . Lin et al ${ }^{18}$ used Raman scattering technique to collect quantitative concentration data. These measurements were used to calculate the mixture fraction at various $x / D$ locations downstream of the injector.

\section{Grid and numerical choices}

In this study, two grids are used a RANS grid for RANS simulations and a multi-scale grid for multi-scale simulations. The RANS contains about 6 million cells with the jet region at a finer resolution and a boundary layer grid starting at $\boldsymbol{y}^{+} \sim 10$. The multi-scale grid contains 29 million cells with the extra cells mainly in the jet and wall regions. Figure 1 shows the lower part of the computational domain and boundary conditions. Figure 2 shows a center cut with the adapted grid areas near the injector. 


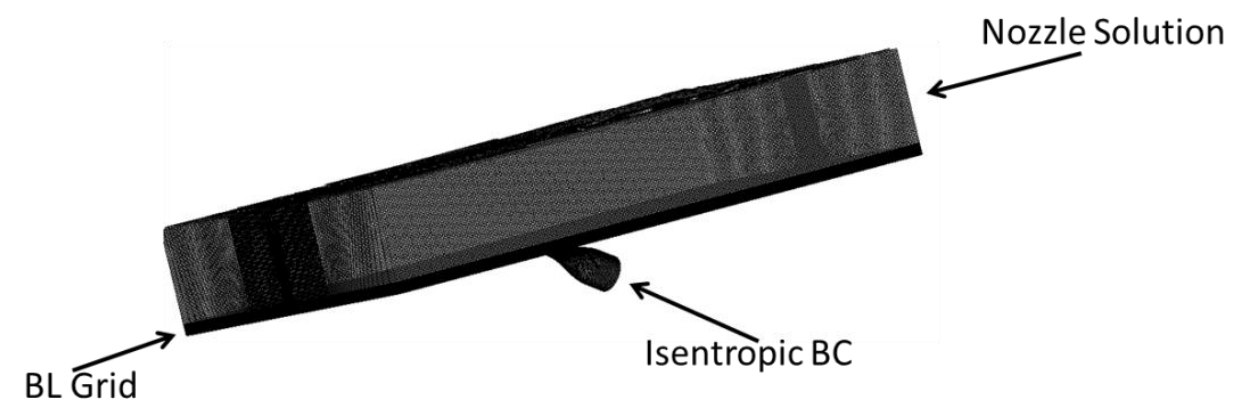

Figure 1. Lower computational domain and boundary conditions.

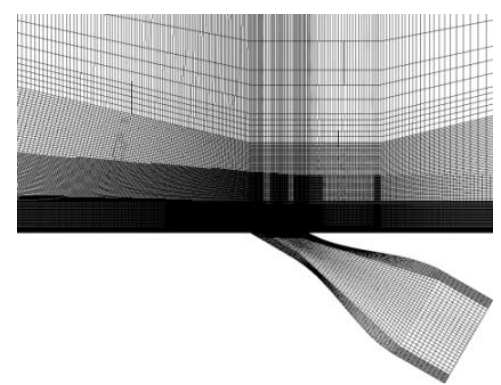

Figure 2. Center plane cut for multi-scale grid. Finer grid is at the jet location and near the wall.

The inlet to the computational domain was taken from a RANS solution of a separate simulation of the nozzle. The full width and height of the wind tunnel was used in the computation. The injector geometry was also representative of that used in the experiment. The normal injection cases used for comparison from a previous study contain 600,000 cells for RANS and 27 million for multi-scale ${ }^{19}$. Boundary conditions and geometrical information of the simulation are shown in Table 1.

Table 1. Flow and geometric conditions for Ethylene injection.

\begin{tabular}{c|c}
\hline Parameters & Values \\
\hline$P_{0}$ (freestream) & $244 \mathrm{KPa}$ \\
$T_{0}$ (freestream) & $300 \mathrm{~K}$ \\
$M$ (freestream) & 2 \\
$P_{0}$ (injectant) & $127.5 \mathrm{KPa}$ \\
$T_{0}$ (injectant) & $322 \mathrm{~K}$ \\
$D$ & $4.8 \mathrm{~mm}$ \\
$q$ & 0.5 \\
Injectant angle $(\theta)$ & $30^{\circ}$ \\
\hline
\end{tabular}

The cases used in this study are RANS and multi-scale simulations with and without the the adaptive $\boldsymbol{S} \boldsymbol{c}_{\boldsymbol{t}}$ approach for inclined 30 degree injection. Additionally, previous 90 degree injection multi-scale case using the fine grid and RANS are used for comparison ${ }^{19}$. Table 2 gives a summary of the cases and their description to facilitate reference to them in the results section. 
Table 2. Description of simulation cases discussed in this effort.

\begin{tabular}{c|cccc}
\hline Case & $\begin{array}{c}\text { Grid } \\
\text { resolution }\end{array}$ & $\begin{array}{c}\text { Turbulence } \\
\text { treatment }\end{array}$ & $S c_{t}$ & angle \\
\hline Previous & & RANS-SST & 0.7 & 90 \\
1 & $600 \mathrm{~K}$ & Multi-scale-SST & 0.7 & 90 \\
2 & $27000 \mathrm{~K}$ & RANS-SST & 0.7 & 30 \\
New & & Multi-scale-SST & 0.7 & 30 \\
3 & $6300 \mathrm{~K}$ & Multi-scale-SST & Adaptive & 30 \\
4 & $29000 \mathrm{~K}$ & & & \\
5 & $29000 \mathrm{~K}$ & & & \\
\hline
\end{tabular}

\section{Results and Discussion}

The RANS inclined injection simulations were run with the SST turbulence model ${ }^{2}$ until converged. RANS results at 6.3 million cells were identical to those obtained at much lower resolutions. The multi-scale approach with and without the adaptive turbulent Schmidt number was applied starting with RANS solution interpolated on the multi-scale grid then instantaneous results were collected and averaged once instabilities are statistically converged $^{19,28}$. Results with the multi-scale model without the adaptive $S c_{t}$ approach along with turbulent fluctuations and vorticity are compared to those of a normal 90 degree injection. The predictions of multi-scale alone are then compared to multi-scale with the adaptive approach and RANS results in predicting experimental measurements. To avoid confusion the term "multi-scale" will refer to multi-scale without the adaptive turbulent Schmidt number approach while the term "adaptive" will be used with multi-scale combined with the adaptive approach.

\section{A. Fuel mixing dynamics in inclined versus normal injection}

Fuel mass fractions are quantitatively compared to the available experimental Raman scattering results at 3 different $x / D$ locations in Figure 3. The fuel concentration is over-predicted at $x / D=5$ by RANS which shows a single horse-show vortex of concentration higher than $70 \%$ and taking up the entirety of the cross section. The multi-scale results show two weaker kidney shaped vortices that are larger than those in the experimental measurements but smaller and weaker than the RANS results. The experimental concentrations at $x / D=5$ are still much weaker than multi-scale even though the latter is conducted with nearly 30 million cells. At $x / D=10$, fuel concentration is over-predicted by both RANS and multi-scale however results are closer to the experiment with the multi-scale approach. There is also a bend in the numerical measurements in both RANS and multi-scale near $y / D=1$ that is not present in experimental results. Penetration heights are also slightly over-predicted with both RANS and multi-scale which corresponds to jet growth faster than that witnessed in the experiment. At $x / D=25$ the predictions of both RANS and multi-scale show a balloon like structure that is more mixed in the case of multi-scale. The results, however, are different from the experiment which shows a single width column attached to the wall. The penetration height is over-predicted due to higher jet growth. Overall, the multi-scale model did not improve the results significantly over RANS especially when looking at similar previous results with the 90 degree injection. ${ }^{19}$ 

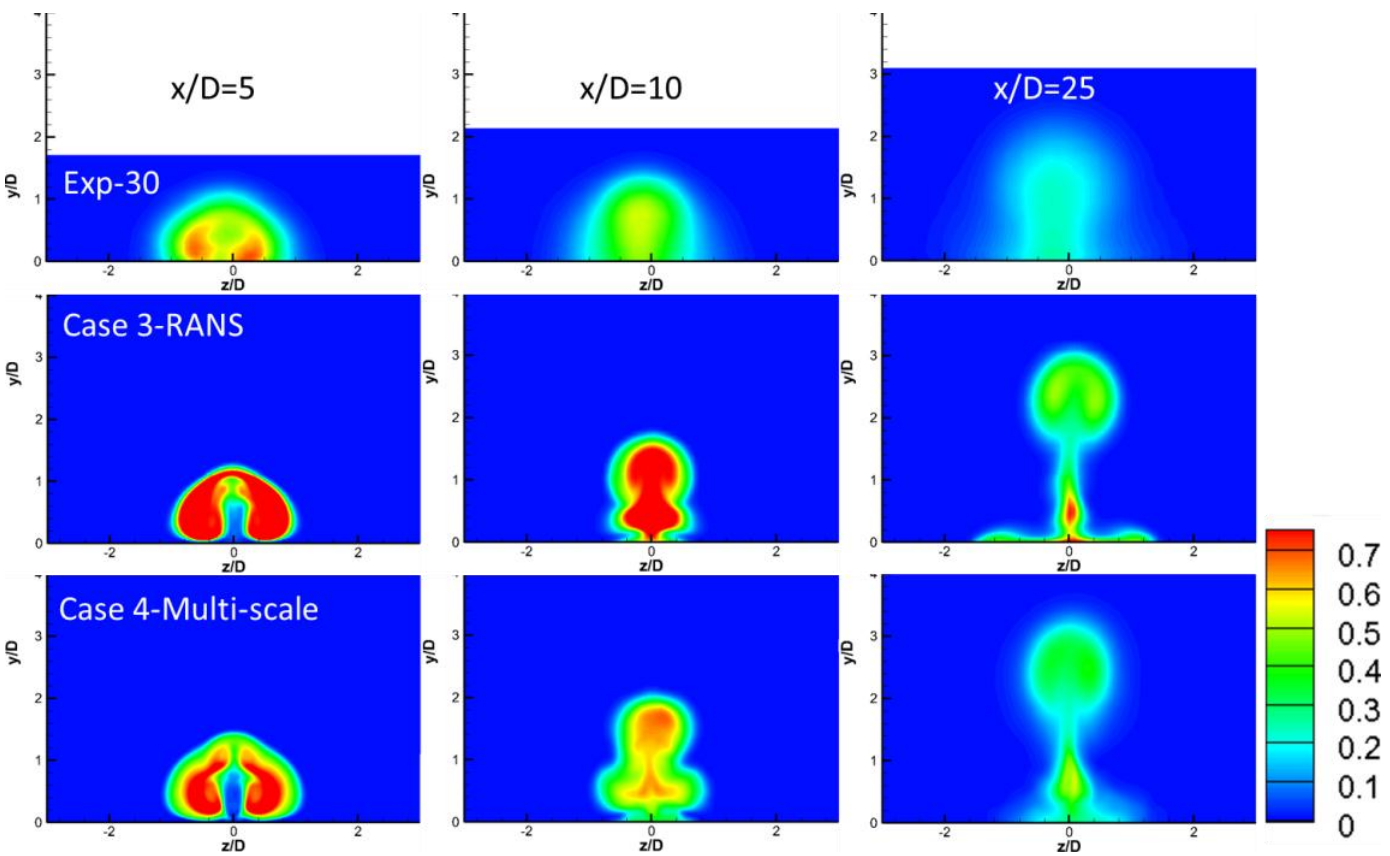

Figure 3. Fuel mass fraction predictions for RANS and multi-scale without the adaptive approach compared to experimental Raman scattering at 3 different axial locations. 30 degree injection.

In a previous study, the multi-scale approach alone was used to simulate normal Ethylene injection in supersonic crossflow $^{19}$. Results showed significant improvement over RANS when compared to the experiment Raman scattering measurements of Lin et al ${ }^{18,37}$. Results of that study are shown in Figure 4 with a grid of 27 million cells.
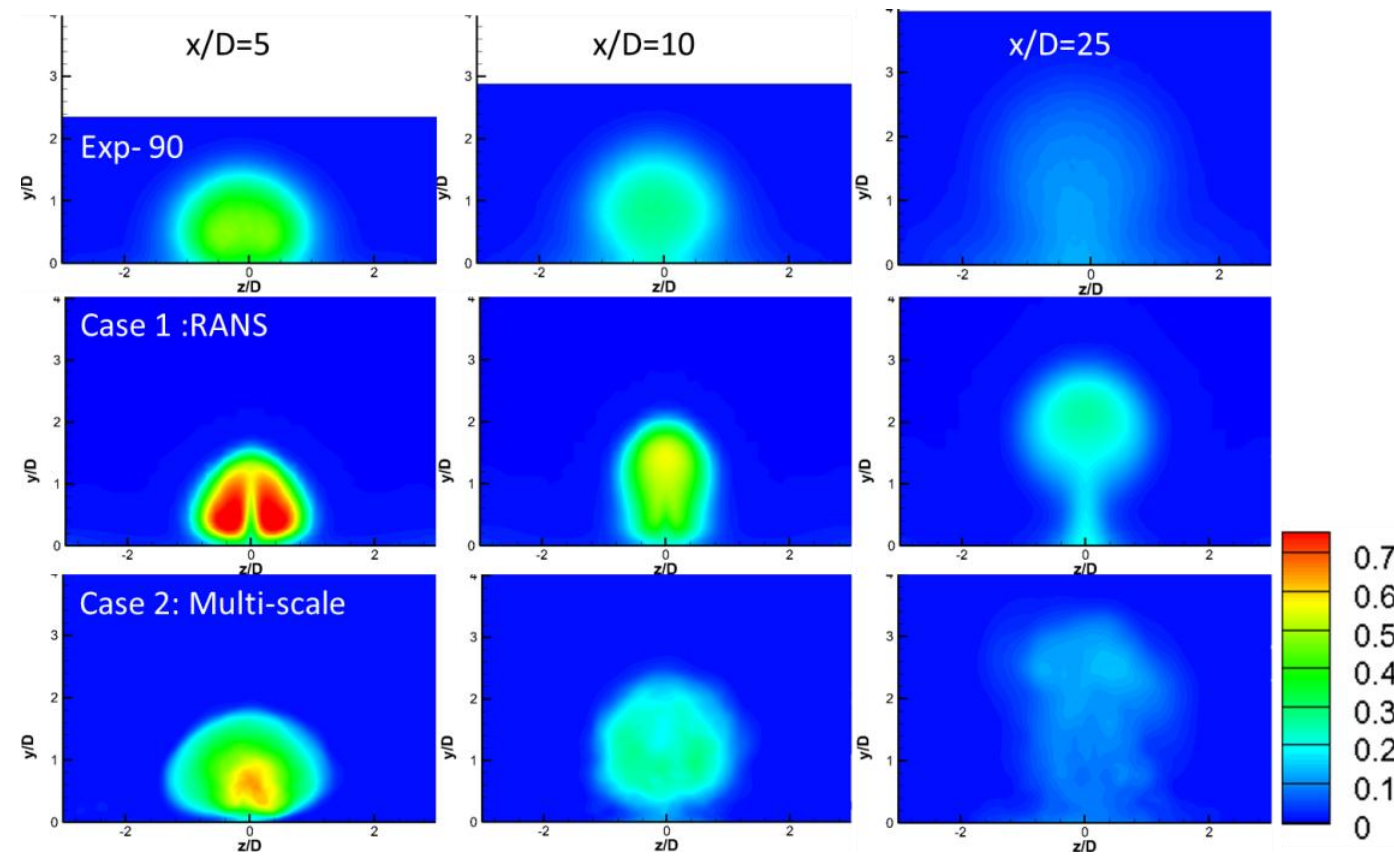

Figure 4. Fuel mass fraction predictions for experimental, RANS and multi-scale without the adaptive approach. 90 degree injection angle from Hassan et al. ${ }^{19}$

At $x / D=5$, the penetration height is well predicted for both RANS and multi-scale. RANS results have a "heart shaped" fuel core with two large kidney vortices that are not present in the experiment. Multi-scale results on the 
other hand show a flatter core with a single vortex that is closer to the experimental measurements. For $x / D=10$, The RANS solution is oval shaped with higher fuel concentration than the experiment. The multi-scale has a circular cross section with concentrations similar to that of the experiment. For $x / D=25$, RANS results show fuel concentration higher than the experiment with a balloon-like shape. The multi-scale has similar shape and concentration to the experimental measurements. Overall, there is a significant improvement in the results when multi-scale is used with normal injection. Results shown in Figure 3 for the inclined injection do not show such an improvement. It is therefore of value to compare normal and inclined injections in terms of turbulent fluctuations.

The dynamics of normal injection differ from those of inclined injection and thus affect the turbulent fluctuations occurring in the flow. In normal injection, violent interactions between the jet and crossflow causes plume breakup and separation between the jet and crossflow. This leads to large scale structures that originate in the near field and have a greater effect in determining the overall shape of the mean flow. Large scale mixing occurs at a lower wave length therefore it is a major factor in mixing. Inclined injection is less violent. There is no turbulent breakup of the fuel plume. Instead fluctuations originate from the edge of the jet near the bow shock. Fluctuations occur more locally and at a higher wave length. Therefore a much finer grid/time scale is needed to resolve important turbulent structures.

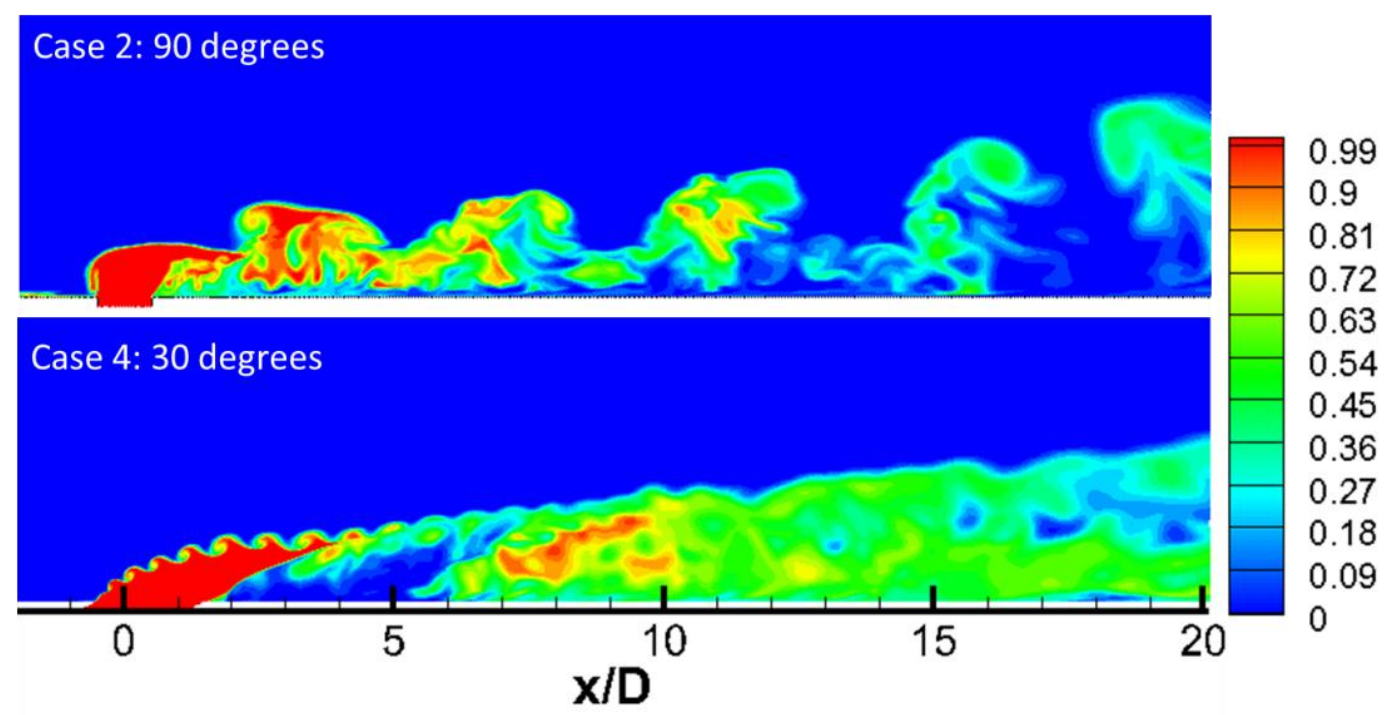

Figure 5. Instantaneous fuel mass fraction snapshots on the center plane for multi-scale approach for both 90 and 30 degree angles.

Figure 5 shows instantaneous snapshots of fuel mass fractions on the center plane for both 90 and 30 degree injections using the multi-scale model. In the near field, violent breakup occurs for the 90 degree case with the fuel plume disintegrating into small fuel packets and sheets. The plume in the 30 degree case is largely intact with small Kelvin-Helmholtz like instabilities near the bow shock where the interaction between jet and crossflow occurs. Further down the field, the entire flow is comprised of large turbulent structures that contain smaller fuel rich vortices that are carried along with them. In the case of 30 degree, the flow has a predetermined background shape in which there are small perturbations and small fuel rich spots that are carried along with the flow. 


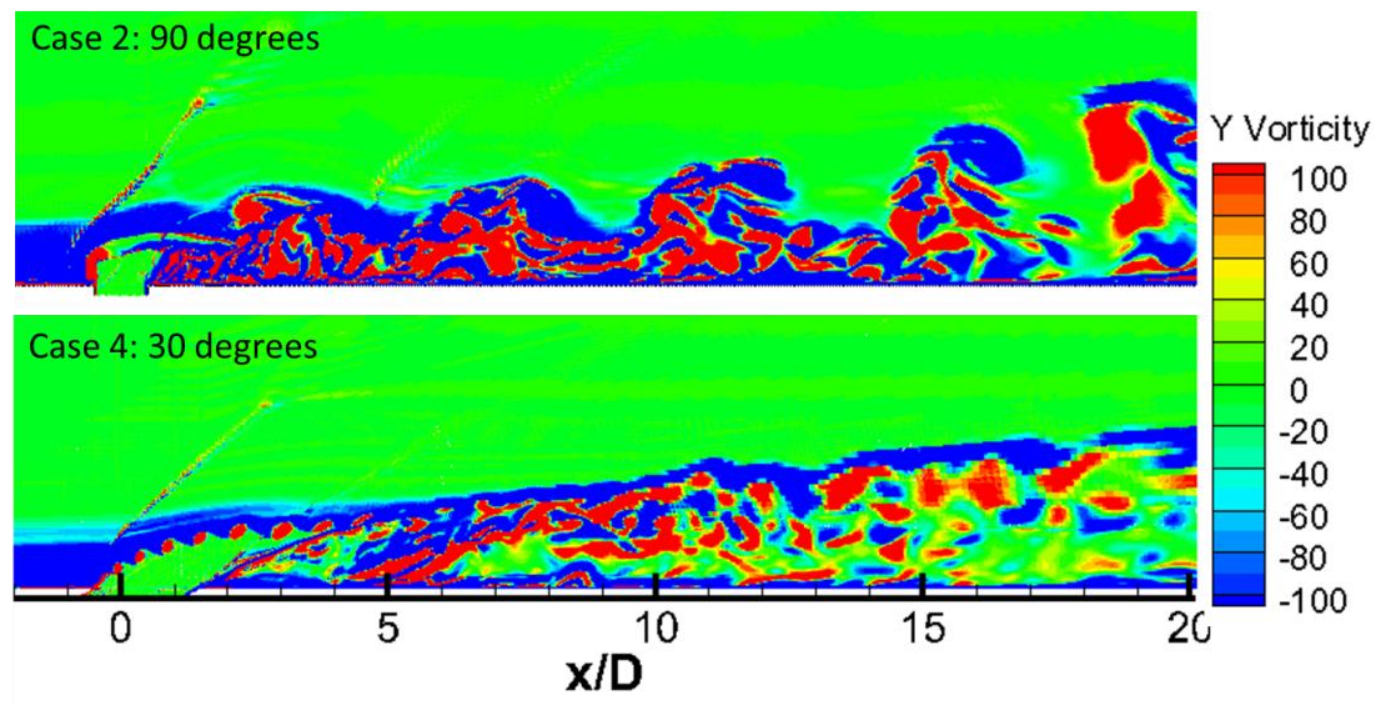

Figure 6. Instantaneous vorticity snapshots on the center plane for multi-scale approach for both 90 and 30 degree angles. . Maximum and minimum limited to 100, -100

The same conclusions can be drawn by looking at the instantaneous vorticity snapshots in Figure 6 . In the case of 90 degrees, the vorticity changes very rapidly from positive to negative and vortical structures are wrapped around each others that the entire flow is comprised of vortical structures. The size of the vortices increases downstream and takes up the entire region where fuel is present. In the case of 30 degrees, vorticity of positive and negative values are smaller and scattered inside the jet. They do not dictate the shape of the jet unlike the case of 90 degree injection. There is a sheet of negative vorticity on the outside boundary of the jet containing all other flow structures. On the inside of the jet scattered vortices appear in the background flow and are carried along with it. Overall the 30 degree injection contains smaller locally concentrated vortices and therefore requires resolving the flow in much more detail than the normal injection.

\section{B. Multi-scale results with and without the adaptive approach}

The adaptive turbulent Schmidt number approach was developed and tested with different grid resolutions for the 90 degree case by Hassan et $\mathrm{al}^{28}$. The turbulent Schmidt number is calculated based on the resolved portion of the flow and is used in the sub-filter RANS model. It was developed to work best in cases where high frequency fluctuations are not readily available from the simulation and those larger ones available are representative of the mixing process. The case of the 30 degree injection is a good candidate because most of the turbulent mixing is carried by smaller vortices that are harder to resolve. Therefore the modeled portion of the flow has a significant effect on the overall turbulent diffusion.

To evaluate the capability of RANS and multi-scale models, first the average fuel mass fraction on the center plane of the injector is collected and shown in grey scale in Figure 7 to compare with the mean intensity of processed PLIF images obtained experimentally by Lin et al. ${ }^{18,37}$ It should be noted that the mean intensity images do not have scaling information, therefore, no scale is displayed. 


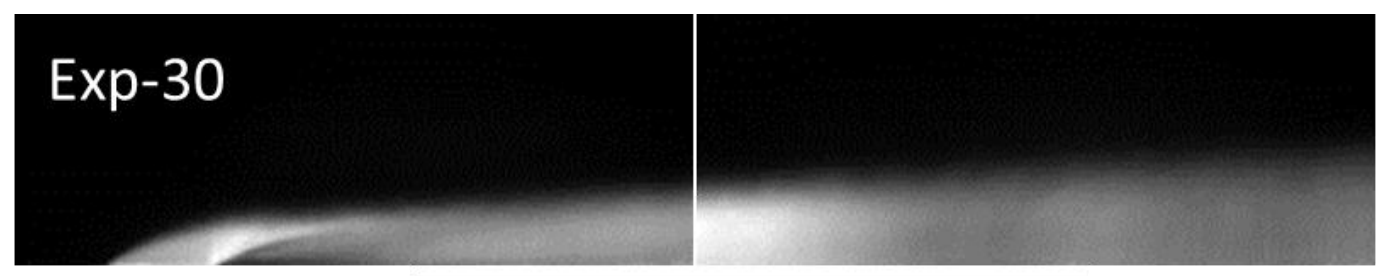

\section{Case 3-RANS}

\section{Case 4-Multi-scale}

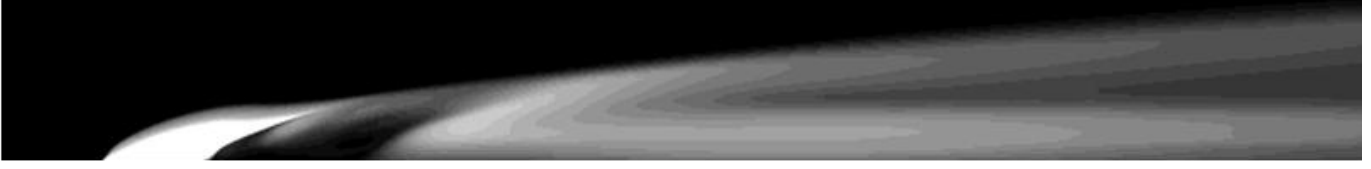

\section{Case 5-Adaptive}

Figure 7. Mean intensity of PLIF images (2 separate images taken) compared to fuel mass fraction contours in grey scale of RANS and multi-scale models.

Despite different grid resolutions, the multi-scale and RANS results have similar jet structure. The intensity of fuel concentration is lower in the case of multi-scale than RANS due to higher degree of mixing. Experimental results show significantly higher turbulent mixing with slower jet growth than either RANS or multi-scale. Below the fuel plume there is a fuel lean pocket that is more pronounced in the RANS and multi-scale results than the experiment because fuel is able to diffuse there with the higher turbulent mixing. In the far field there is also a slender fuel lean pocket in the center of the jet in RANS and multi-scale that is not present in experimental mean PLIF images. Overall multi-scale results are slightly more mixed than RANS while still not significantly closer to the experiment. With the adaptive approach, similar to the experiment, fuel lean pocket under the plume is smeared due to high turbulent mixing. The jet growth and penetration heights are well predicted with the jet only slightly larger than the experiment. Fuel concentration in the far field is also slightly under-predicted with the adaptive approach. Overall the adaptive results are very good compared to the experiment and are a significant improvement over RANS and multi-scale alone.

In order to qualitatively check the resolved turbulent fluctuations in multi-scale models, the variance of the fuel mass fraction is compared to the variance of the intensity of the PLIF images. The results of the comparison are plotted in greyscale in Figure 8. Scaling information is not shown because it is not present with the experimental results. Naturally, the RANS results show an insignificant variance because no variance is captured as the high eddy viscosity dampens turbulent. The multi-scale variance shows some similarity to the experiment especially near the jet exit. 


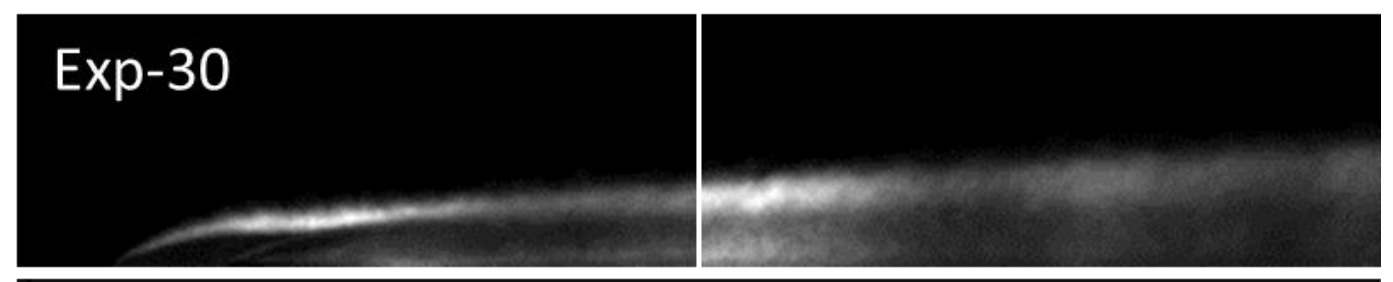

Case 3-RANS

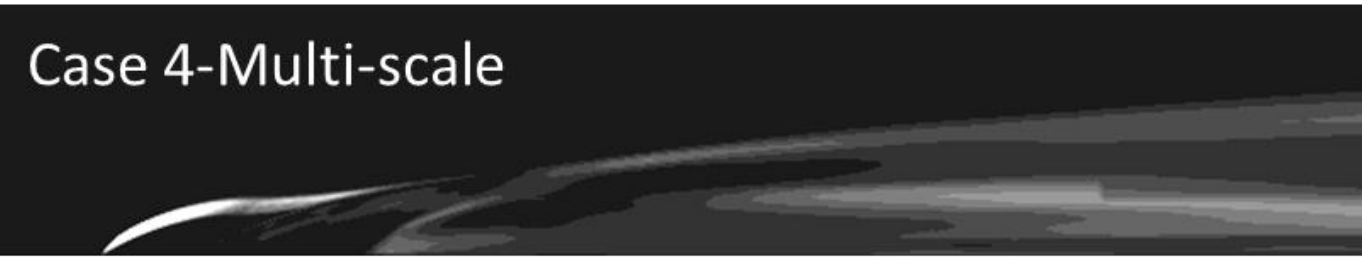

Case 5-Adaptive

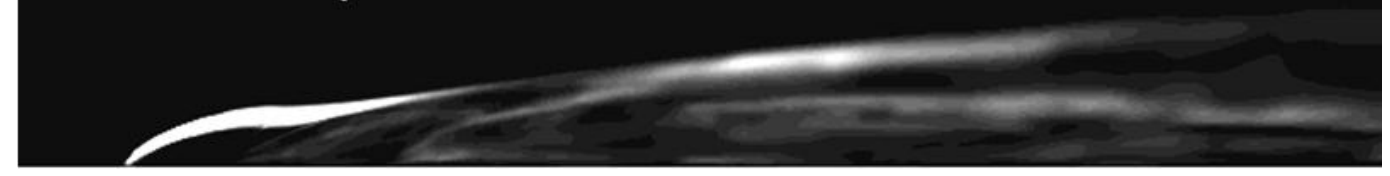

Figure 8. Variance of intensity of PLIF images( 2 separate images taken) compared to fuel mass fraction variance contours in grey scale of RANS and multi-scale models.

At the top edge of the fuel plume there is an area of high variance which corresponds to the fluctuations originating below the bow shock. This area is present in both experimental and multi-scale plots. Following the upper edge of the jet there is a decrease in variance that is present in both multi-scale and experiment, however, it is more pronounced in the multi-scale plot. The variance then increases and decreases again as we move to the far field. This behavior is also present in the multi-scale results. Near the wall there are also similar results in the near field in both RANS and multi-scale, however, as we move away from the jet, there is a high variance region in the multi-scale results that is not present in the experiment. Overall the multi-scale captures similar variance dynamics as the experiment except in the far field and near the center of the jet.

The adaptive approach displays the same variance characteristics as those obtained from PLIF images. Following the upper jet boundary there is a higher variance region that decreases and increases in intensity. The entire length of the upper jet boundary is well captured by the simulation and is in much better agreement than the multi-scale results alone. The inside of the jet shows lower variance in the fuel plume and the fuel lean pocket is in agreement with the experiment. There is some deviation in variance in the far field below the jet boundary. Those disagreements are far less obvious than those shown with the multi-scale alone. Overall the variance obtained using the adaptive approach is superior to those obtained without it when compared to the experiment. 

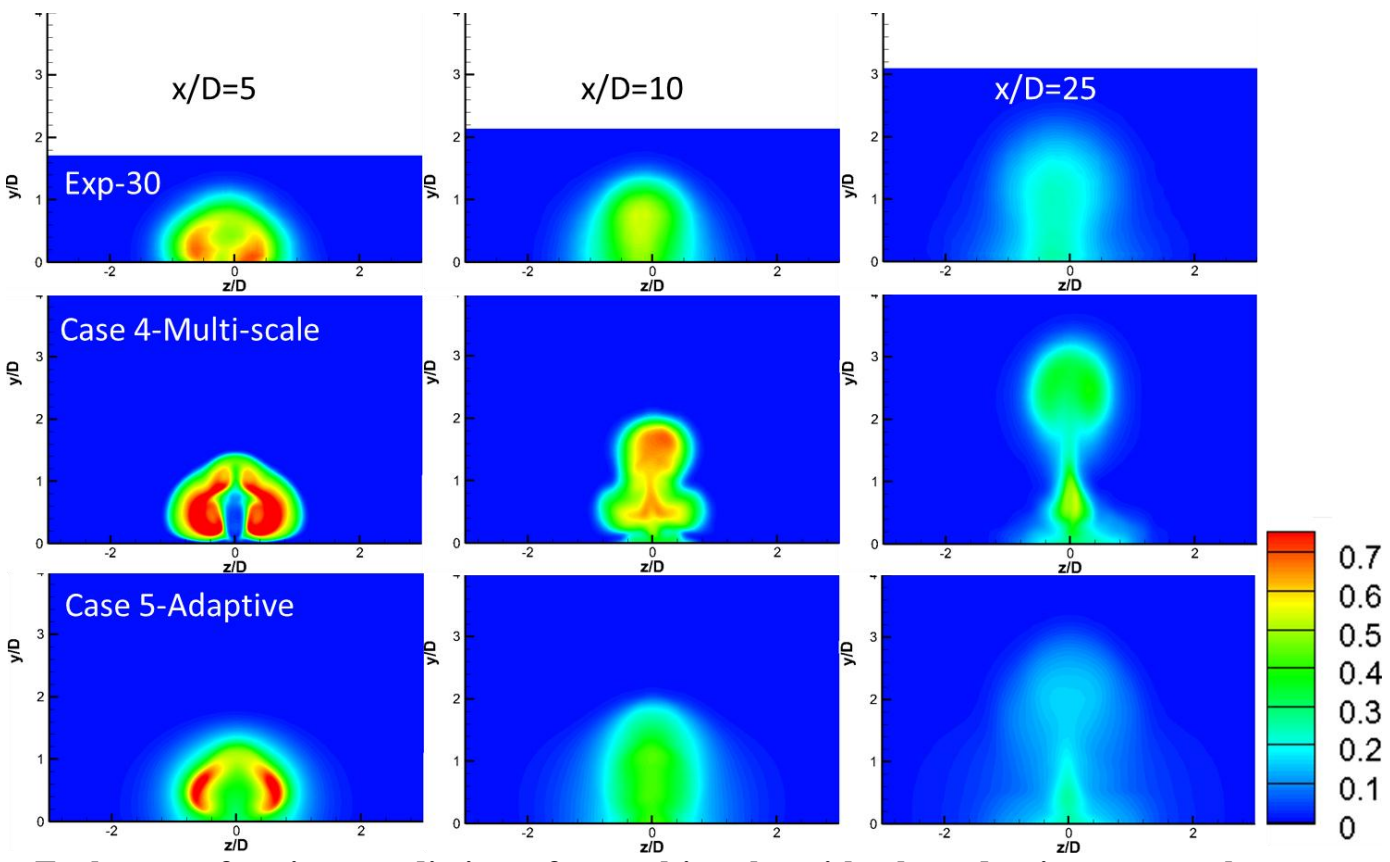

Figure 9. Fuel mass fraction predictions for multi-scale with the adaptive approach compared to experimental Raman scattering at 3 different axial locations.

Experimental Raman scattering fuel concentration results are compared to the adaptive approach at 3 axial locations. Fuel concentration is slightly over predicted at $x / D=5$ with the overall structure of the fuel core similar to that of the experiment. Penetration height and width are well predicted. At $x / D=10$, fuel concentration is well predicted with similar structure however penetration height is slightly over predicted. There is no bend in the numerical results unlike multi-scale alone. At $x / D=25$, the predictions of the adaptive approach are of similar structure to the experimental measurements, however, penetration height is over predicted and higher concentration fuel core is thinner in the center. There is no balloon like structure unlike multi-scale alone.

Adaptive turbulent Schmidt number contours for 3 axial locations are plotted in Figure 10. In most areas of interest the value of turbulent Schmidt number is less than 0.7. There are areas however with high values of $S c_{t}$ corresponding to low turbulent diffusion. Overall, the value of $S c_{t}$ varies dramatically throughout the flow. There is symmetry around $z / D=0$ in $S c_{t}$ contours which is a sign that good quality fluctuations have been collected from the flow field ${ }^{28}$. Symmetry is more apparent in the near field, however, at $x / D=25$ there is still overall symmetry in $S c_{t}$ contours.
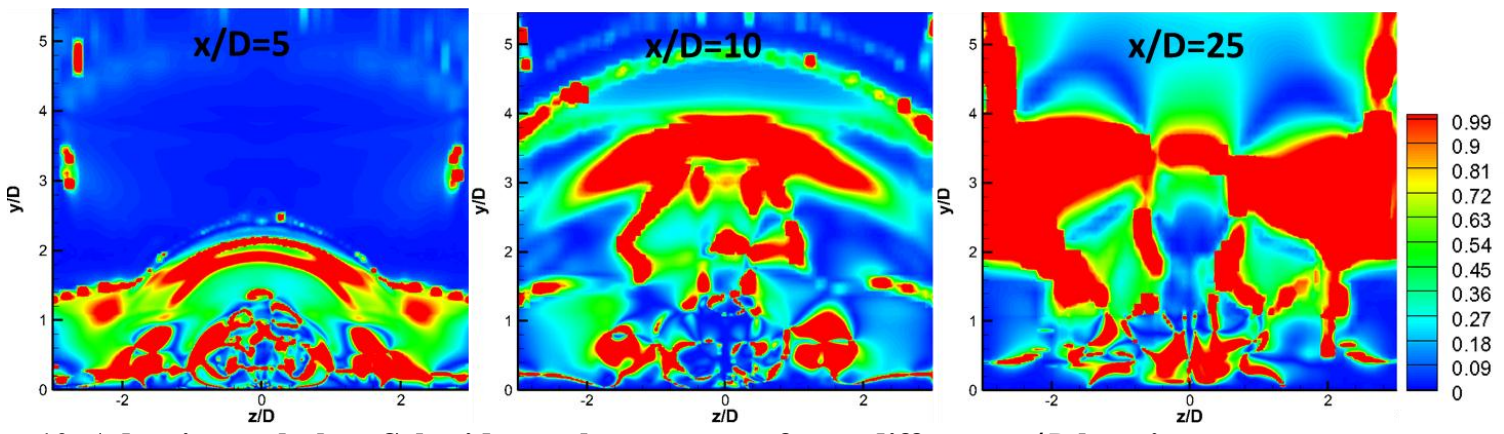

Figure 10. Adaptive turbulent Schmidt number contours for at different $x / D$ locations. 


\section{Summary and Conclusions}

An inclined 30 degree injection case is successfully simulated with the multi-scale model both with and without the adaptive approach. A large grid with 29 million cells is used to resolve and analyze more turbulent structures. When compared to experimental fuel concentration, multi-scale without the adaptive approach showed minor improvement over RANS. Unlike a previous study with normal 90 degree injection, multi-scale alone did not significantly improve the results. Comparison to the 90 degree injection case reveals smaller turbulent fluctuations that are of higher frequency than the 90 degree case. Vortical structures in the 30 degree case are scattered in a back ground representing the main flow. In the 90 degree case vortical structures were larger and more complex due to violent interaction between the jet and cross flow. Smaller scattered vortices in the 30 degree case therefore require more accurate sub-filter model of the turbulent mixing to avoid prohibitively expensive simulations. The adaptive turbulent Schmidt number approach is used to improve the prediction of turbulent diffusion in the sub-filter RANS model based on the resolved portion of the flow. When the adaptive approach is employed, the results improve significantly. Mean and variance fuel concentration predictions compare favorably with experimental measurements and are far superior to either RANS or multi-scale without adaptive Scmidt number.

\section{Acknowledgements}

Ez A Hassan would like to acknowledge the SMART program for their support. Portions of the computations were performed on AFRL high performance computing clusters.

\section{References}

1. Tam, C.-J., R.A. Baurle, and M.R. Gruber. Numerical Study of Jet Injection into a Supersonic Crossflow. in 35th AIAA/ASME/SAE/ASEE Joint Propulsion Conference and Exhibit. 1999. Los Angeles, california.

2. Menter, F.R., Zonal two equations $k$-w turbulence models for aerodynamic flows. AIAA, 1993. 93(2909).

3. Wilcox, D.C., Simulation of Transition With a Two-Equation Turbulence Model. AIAA Journal, 1994. 32: p. $247-254$.

4. Gruber, M.R., Nejad, A S., J. C. Dutton, An Experimental Investigation of Transverse Injection from Circular and Elliptical Nozzles into supersonic Crossflow. Wright Lab Technical Report, 1996. WL-TR-96-2102.

5. Gruber, M.R., A.S. Nejad, T.H. Chen, and J.C. Dutton, Large structure convection velocity measurements in compressible transverse injection flowfields. Experiments in Fluids, 1997. 22 (1997): p. 397-407.

6. Gruber, M.R., A.S. Nejad, T.H. Chen, and J.C. Dutton, Compressibility Effects in Supersonic Transverse Injection Flowfields. Physics of Fluids, 1997. 9(5): p. 1448-1461.

7. Palekar, A., C.R. Truman, and P. Vorobieff, Prediction of Transverse Injection of a Sonic Jet in a Supersonic Crossflow, in 36th AIAA Plasmadynamics on Lasers Conference. 2005: Toronto, Ontario Canada.

8. $\quad$ Maddalena, L., T.L. Campioli, and J.A. Schetz, Experimental and Computational Investigations of Light-Gas Injectors in Mach 4.0 Crossflow. Journal of Propulsion and Power, 2006. 22(5).

9. Nichols, R.H. and R. Tramel, Applications of a Highlt Efficient Numerical Method for Overset Mesh Moving Body Problems. AIAA, 1997. 97-2255.

10. Sinha, N., S. Dash, and N. Chidambaram, A perspective on the Simulation of Cavity Aeroacoustics. AIAA, 1998. 980286.

11. Spalart, P., W. Jou, and M. Strelets, Comments on the Feasibility of LES for Wings and ona Hybrid RANS/LES Approach, ed. C. Liu and Z. Liu. 1997: Greyden Press, Columbus, OH.

12. Kawai, S. and S.K. Lele, Large-Eddy Simulation of Jet Mixing in Supersonic Crossflows. AIAA Journal, 2010. 48(9): p. 2063-2083.

13. Santiago, J.G. and J.C. Dutton, Velocity Measurements of a Jet Injected into a Supersonic Crossflow. Journal of Propulsion and Power, 1997. 11(2): p. 315-323.

14. Peterson, D.M., P.K. Subbareddy, and G.V. Candler, Assessment of Synthetic Inflow Generation for Simulating Injection into Supersonic Crossflow, in 14th AIAA/AHI Space Planes and Hypersonic Systems and Technologies Conference. 2006.

15. Boles, J.A., Hybrid Large-Eddy Simulation/Reynolds-Averaged Navier-Stokes Methods and Predictions for Various High-Speed Flows, in Aerospace Engineering. 2009, North Carolina State University: Raleigh, NC.

16. Boles, J.A. and J.R. Edwards, Hybrid LES/RANS Simulation of Transverse Sonic Injection into a Mach 2 Flow, in 46th AIAA Aerospace Sciences Meeting and Exhibit. 2008: Reno, Nevada.

17. Boles, J.A., J.R. Edwards, and R.A. Baurle, Large-Eddy/Reynolds-Averaged Navier-Stokes Simulations of Sonic Injection into Mach 2 Crossflow. AIAA Journal, 2010. 48(7): p. 1444-1456.

18. Lin, K.-C., M. Ryan, C. Carter, M. Gruber, and C. Raffoul, Scalability of Ethylene Gaseous Jets for Fueling HighSpeed Air-Breathing Combustors, in 47th AIAA Aerospace Sciences Meeting and Exhibit. 2009: Orlando, Florida.

16

American Institute of Aeronautics and Astronautics 
19. Hassan, E., H. Aono, J. Boles, D. Davis, and W. Shyy. Multi-Scale Turbulence Model in Simulation of Supersonic Crossflow. in 49th AIAA Aerospace Sciences Meeting including the New Horizon Forum and Aerospace Exposition 2011. Orlando, Florida.

20. Peterson, D.M. and G.V. Candler, Supersonic Combustor Fuel Injection Simulation Using a Hybrid RANS/LES Approach, in 48th AIAA Aerospace Sciences Meeting Including the New Horizon Forum and Aerospace Exposition. 2010: Orlando, Florida.

21. Reynolds, A.J., The Prediction of Turbulent Prandtl and Schmidt Numbers. Int. J. Heat and Mass Transfer, 1975. 18: p. 1055-1069.

22. Negano, Y., M. Kondoh, and M.Shimada, Multiple time-scale turbulence model for wall and homogenous shear flows based on direct numerical simulation. Int. J. Heat Fluid Flow, 1997. 18(4): p. 347-359.

23. Sommer, T.P., R.M.C. So, and H.S. Zhang, Near-wall variable Prandtl-Number turbulence model for compressible flows. AIAA Journal, 1993. 31(1): p. 27-35.

24. Guo, Y., G. He, A.T. Hsu, A. Brankovic, S. Syed, and N.S. Liu, The Development of a Variable Schmidt Number Model for Jet-in-Crossflow Using Genetic Algorithms. AIAA Paper, 1999(99-0671).

25. Kenzakowski, D.C., J. Papp, and S.M. Dash, Evaluation of Advanced Turbulence Models and Variable Prandtl/Schmidt Number Methodology for Propulsive Flows. AIAA Paper, 2000(2000-0885).

26. Brinckman, K.W., D.C. Kenzakowsi, and S.M. Dash, Progress in Practical scalar Fluctiation Modeling for High Speed Aeropropulsive Flows. AIAA Paper, 2005(2005-0508).

27. Keislter, P.G., A Variable Turbulent Prandtl and Schmidt Number Model Study for Scramjet Applications, in Mechanical and Aerospace Engineering. 2009, North Carolina State University: Raleigh, North Carolina.

28. Hassan, E., H. Aono, J. Boles, D. Davis, and W. Shyy, Adaptive Turbulent Schmidt number approach for Multi-Scale Simulation of Supersonic Crossflow in 20th AIAA Computational Fluid Dynamics Conference. 2011: Honolulu, Hawaii.

29. Luke, E.A. and P. Cinnella, Numerical simulations of mixtures of fluids using upwind algorithms. Computers \& Fluids, 2007. 10: p. 1547-1566.

30. Luke, E.A. and T. George, Loci: A rule-based framework for parallel multidisciplinary simulation synthesis. Journal of Functional Programming, 2005. 15(3): p. 477-502.

31. Roe, P.L., Approximate Riemann solvers parameter vectors and difference schemes. Journal of Computational Physics, 1981. 43: p. 357-372.

32. Germano, M., From RANS to DNS: Towards a Bridging Model. ERCOFTAC Series, 1999. 7: p. 225-236.

33. Johansen, S.T., J. Wu, and W. Shyy, Filter-based unsteady RANS computations. International Journal of Heat and Fluid Flow, 2004. 25: p. 10-21.

34. Nichols, R.H. and C.C. Nelson, Application of Hybrid RANS/LES Turbulence Models, in 41st Aerospace Science Meeting and Exhibit. 2003: Reno, Nevada.

35. Garnier, E., M. Mossi, P. Sagaut, P. Comte, and M. Deville, On the Use of Shock-Capturing Schemes for Large-Eddy Simulation. Journal of Computational Physics, 1999. 153(2): p. 273-311.

36. Mossi, M. and P. Sagaut, Numerical investigation of fully developed channel flow using shock-capturing schemes. Computers \& Fluids, 2003. 32(2): p. 249-274.

37. Lin, k.-C., M. Ryan, C. Carter, M. Gruber, and C. Raffoul, Raman Scattering Measurements of Gaseous Ethylene Jets in Mach 2 Supersonic Crossflow. Journal of propulsion and power 2010. 26: p. 503-513. 